\title{
Reduction of Nitroarenes to Aromatic Amines with Sodium Borohydride in the Presence of Selenium and Actived Carbon
}

\author{
Ke Ying Cai*, Ying Mei Zhou \\ School of Chemistry and Chemical Engineering, Xuzhou Institute of Technology, Xuzhou, \\ Jiangsu 221111, China
}

Received: $3^{\text {rd }}$ May 2015; Revised: $5^{\text {th }}$ October 2015; Accepted: $5^{\text {th }}$ October 2015

\begin{abstract}
A selenium and actived carbon (AC) catalyst has been applied for the selective reduction of nitroarenes to their corresponding amines respectively using sodium borohydride $\left(\mathrm{NaBH}_{4}\right)$ as a reducing source under mild conditions. Under the optimized conditions, efficient and selective reduction of nitroarenes into the corresponding aromatic amines occurred over a recyclable selenium catalyst. The catalyst can be easily recovered after catalytic reaction and readily reused for 4 cycles with consistent activity hence reduces the cost of the catalyst. (C) 2015 BCREC UNDIP. All rights reserved.
\end{abstract}

Keywords: Sodium Borohydride; Reduction; Nitroarenes; Selenium; Amines; Activated Carbon

How to Cite: Cai, K.Y., Zhou, Y.M. (2015). Reduction of Nitroarenes to Aromatic Amines with Sodium Borohydride in the Presence of Selenium and Actived Carbon. Bulletin of Chemical Reaction Engineering \& Catalysis, 10 (3): 275-280. (doi:10.9767/bcrec.10.3.8512.275-280)

Permalink/DOI: http://dx.doi.org/10.9767/bcrec.10.3.8512.275-280

\section{Introduction}

Aromatic amines are important intermediates in organic synthesis, which are widely used in production of medicine, dyes, agrochemical, additive, surfactants, textile assistant and so on. The vast majority of aromatic amines are obtained by the way of reducing nitroarenes. Aromatic amines can be prepared by a great variety of reduction methods. Most of the methods viz. metal/acid reduction, catalytic hydrogenation, electrolytic reduction, metal mediated reduction, homogeneous catalytic transfer hydrogenation, heterogeneous catalytic transfer hydrogenation, etc. are in practice [1]. Nowadays, most large-scale aromatic

* Corresponding Author.

E-mail: caikeying@163.com (K.Y. Cai)

Telp: +86-13852081673, Fax: +86-516-85608300 amines are being produced by catalytic hydrogenation of the corresponding nitro compounds. With a variety of catalyst such as $\mathrm{Pd}, \mathrm{Ni}, \mathrm{Pt}$, $\mathrm{Ag}$, $\mathrm{Co}$ and $\mathrm{Cu}$ [2], the corresponding amines are obtained without the production of waste products. The catalytic hydrogenation is suitable for mass production of amines, but it is less selective and dangerous because of hydrogen gas as reductive agent. The iron/acid reduction has high selectivity, and the reaction condition is mild, but a lot of iron mud from the reduction would pollute environment seriously. The sodium sulfide reduction has mild reaction conditions, but harmful gas and water would be sent out in the reaction. While hydrogen is invariably employed in bulk processing, transfer hydrogenation offers an alternative when pressure equipment and hydrogen handling facilities are unavailable. Hydrazine hydrate, sodium borohydride, lower aliphatic alcohols and formates are typically employed as hydrogen- 
transfer agents [3].

The catalytic transfer hydrogenation (CTH) has many advantages such as mild reaction conditions, high selectivity of anilines, low pollution, so the application in practice gradually increased. During the past decades, the application of $\mathrm{NaBH}_{4}$ as a mild reducing agent has brought about many changes in the reduction of functional groups in organic synthesis. It is known that solely sodium borohydride can not reduce nitroarenes under common conditions unless provided with some catalysts to remove the kinetic barrier of the reduction. Catalysts, like charcoal [4], $\mathrm{Pd}[5], \mathrm{Au}$ [6], $\mathrm{Ag} / \mathrm{SiO}_{2}$ [7], $\mathrm{Ag} /$ porous silicon [8], Raney Ni [9], $\mathrm{CuO} / \gamma-\mathrm{Al}_{2} \mathrm{O}_{3}$ [10], Au/Ag [11] and nano-Ag [12,13], have been used in the reduction.

The cheap nonmetal selenium catalyst possesses both efficiency to activate carbon monoxide and the function of phase-transfer catalysis. What is more, it can be recycled. Substitution of noble metal catalyzed carbonylation and virulent phosgene methods with Se/CO catalytic system to carry out carbonylation reaction, together with applying $\mathrm{Se} / \mathrm{CO} / \mathrm{H}_{2} \mathrm{O}$ to carry out highly selective reduction, has many advantages such as mild reaction conditions, high atomic economy, low cost, benign environment, short synthesis approach and simple operation, which arouse broad attention [14].

Herein, we report convenient methodologies for nitroarene reduction in the presence of selenium and actived carbon (AC) under milder conditions. To the best of our knowledge this is the first report on reduction of nitroarenes with sodium borohydride catalyzed by selenium and AC (Scheme 1).

\section{Materials and Methods}

\subsection{Materials and Instrumentations}

Sodium borohydride and AC was purchased from Tianjin Fucheng. Selenium was purchased from Xingta Chemical Factory. Nitrobenzene was purchased from Shanghai Hanhong. All other chemicals, unless otherwise specified, used in the present work were of analytic grade.

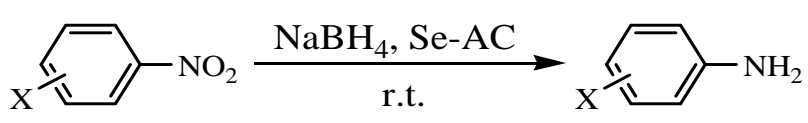

Scheme 1. CTH of nitroarenes to corresponding amines

\subsection{Reduction of Nitroarenes}

Nitroarenes $(10 \mathrm{mmol})$, selenium powder $(0.1 \mathrm{~g}), \mathrm{AC}(0.5 \mathrm{~g})$, ethanol $(12 \mathrm{~mL})$ and water $(4$ $\mathrm{mL}$ ) were added into a $50 \mathrm{~mL}$ two-necked flask fitted with a condensator and a glass stopper. Sodium borohydride $(12 \mathrm{mmol})$ was added to the flask in batches at room temperature with vigorous stirring. The reactions were monitored by Shimadzu GC (Model GC-2014A, FID detector). After the completion of reaction, the air was introduced into the reaction mixture to precipitate selenium. After filtration of selenium and $\mathrm{AC}$, the filtrate was concentrated and dried over anhydrous magnesium sulfate and analyzed. The filtered selenium and $\mathrm{AC}$ cake was washed with ethanol for reuse. The amounts of products were determined by GC using $n-\mathrm{C}_{7} \mathrm{H}_{16}$ as an internal standard and the products were characterized by HewlettPackard GC-MS (Model HP6890/5973).

\section{Results and Discussion}

\subsection{Effect of Selenium and AC}

Initially to search for the optimum reaction condition, nitrobenzene was considered as test substrate. Reduction of nitrobenzene with sodium borohydride was carried out respectively in the absence of a catalyst, the presence of selenium,the presence of $\mathrm{AC}$, and the presence of selenium and $\mathrm{AC}$ to investigate the catalytic effect of selenium and AC. As shown in Table 1, no reaction took place after $2 \mathrm{~h}$ without a catalyst. It is to be noted that the yields of aniline were poor either in the absence of selenium or AC. But when catalyzed by selenium and AC, nitrobenzene was reduced with good yield. The results suggest that the $\mathrm{AC}$ could enhanced the catalytic property of selenium. First, during the reaction selenium was dispersed on $\mathrm{AC}$, which was prone to aggregate without AC. Second,

Table 1. Effect of selenium and AC on the reaction

\begin{tabular}{ccc}
\hline No. & Catalytic system & Yield (\%) \\
\hline 1 & No & 0.0 \\
2 & Selenium & 11.2 \\
3 & AC & 8.8 \\
4 & Selenium and AC & 78.8 \\
\hline
\end{tabular}

Reaction conditions: nitrobenzene (10 mmol), sodium borohydride (12 mmol), selenium $(0.1 \mathrm{~g})$ and $\mathrm{AC}(0.5 \mathrm{~g})$ in ethanol reacting for $2 \mathrm{~h}$ at room temperature. 
AC with large surface area had excellent adsorption ability for organic compounds, which increased the effective contact between the molecules of nitrobenzene and catalyst. Third, the carbon atoms of AC interacted with the terminal oxygen atoms of the nitrobenzene, thereby weakening the $\mathrm{N}-\mathrm{O}$ bonds for effective reduction [15].

Table 2. Effect of solvent on the yield of aniline

\begin{tabular}{clc}
\hline Entry & \multicolumn{1}{c}{ Solvent } & Yield (\%) \\
\hline 1 & DMF & 0 \\
2 & $V($ DMF $): V($ water $)=3: 1$ & 20.1 \\
3 & methanol & 38.8 \\
4 & $V($ methanol): $V$ (water) $=5: 1$ & 65.7 \\
5 & $V($ methanol): $V($ water $)=3: 1$ & 69.6 \\
6 & ethanol & 78.8 \\
7 & $V($ ethanol): $V($ water $)=10: 1$ & 93.1 \\
8 & $V($ ethanol): $V($ water $)=5: 1$ & 94.6 \\
9 & $V($ ethanol): $V($ water $)=3: 1$ & 98.3 \\
10 & $V($ ethanol): $V($ water $)=2: 1$ & 84.7 \\
\hline
\end{tabular}

Reaction conditions: nitrobenzene $(10 \mathrm{mmol})$, sodium borohydride (12 mmol), selenium $0.1 \mathrm{~g}$ and $\mathrm{AC} 0.5 \mathrm{~g}$ in solvent reacting for $2 \mathrm{~h}$ at room temperature.

\subsection{Effect of Solvents}

From early experiments, it was found that solvents have a considerable effect on the reaction. The effect of different solvents on the reaction is shown in Table 2. The polar aprotic solvent such as dimethylformamide (DMF) is not suitable for the reaction (entry 1), while the protic solvents such as methanol and ethanol are good solvents for the reaction. Firstly, nitrobenzene, sodium borohydride and $\mathrm{Se}^{2-}$ produced in the process of reaction all can be soluble in methanol or ethanol, the reaction can be carried out in a homogeneous phase. Secondly, the reaction need hydrogen donor such as methanol, ethanol and water. Water can promote the dissolution of sodium borohydride and $\mathrm{Se}^{2-}$, so a little water added to the system is fa-

Table 3. Recylability of selenium and AC for the reduction of nitrobenzene

\begin{tabular}{ccc}
\hline Entry & Cycle & Yield (\%) \\
\hline 1 & 0 & 98.3 \\
2 & 1 & 99.1 \\
3 & 2 & 98.7 \\
4 & 3 & 98.8 \\
5 & 4 & 98.1 \\
\hline
\end{tabular}

Reaction conditions: nitrobenzene (10 mmol), sodium borohydride $(12 \mathrm{mmol})$, selenium $0.1 \mathrm{~g}$ and $\mathrm{AC} 0.5 \mathrm{~g}$ in ethanol/water reacting for $2 \mathrm{~h}$ at room temperature.
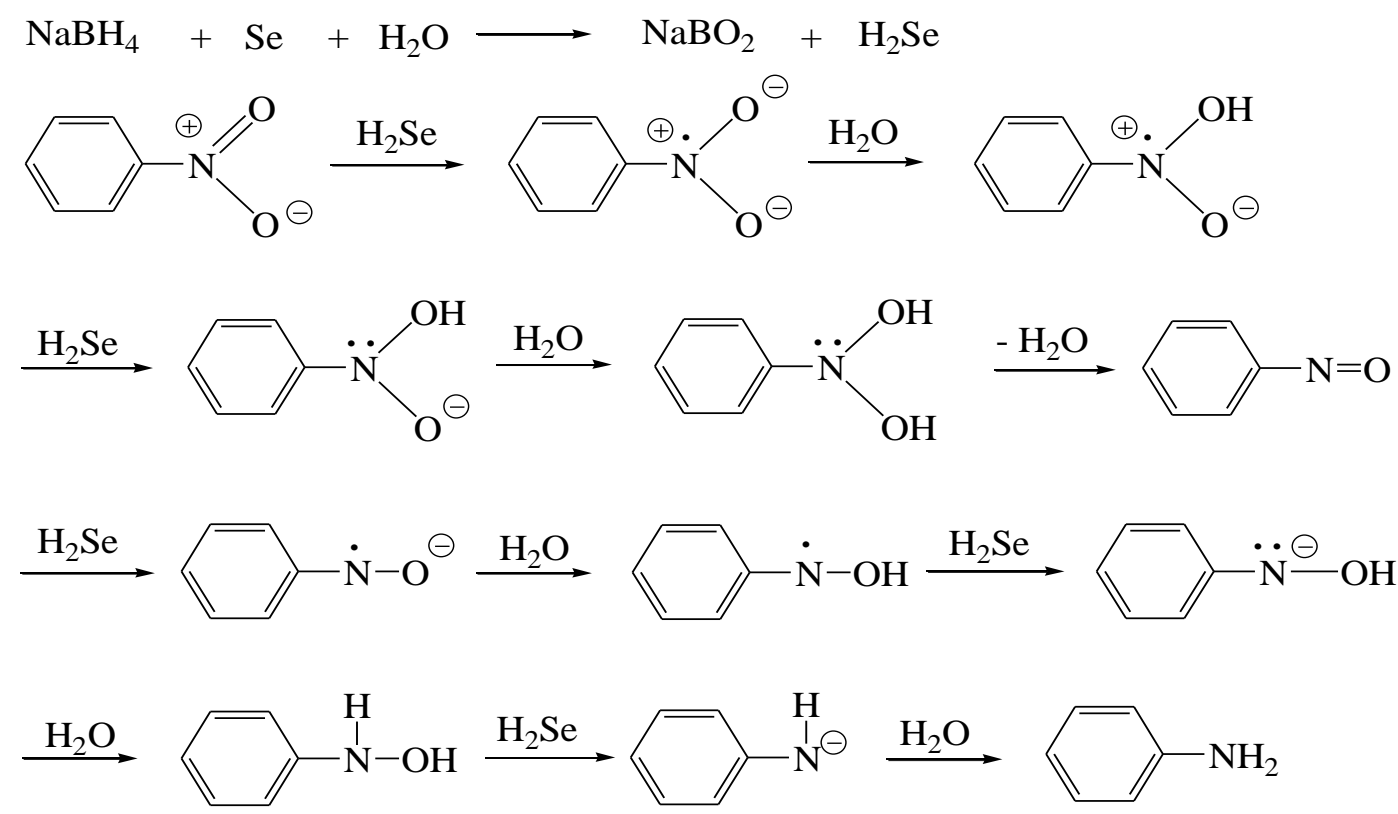

Scheme 2. A possible reaction pathway for the reduction 
Table 4. The reduction of nitroarenes with sodium borohydride over selenium and AC

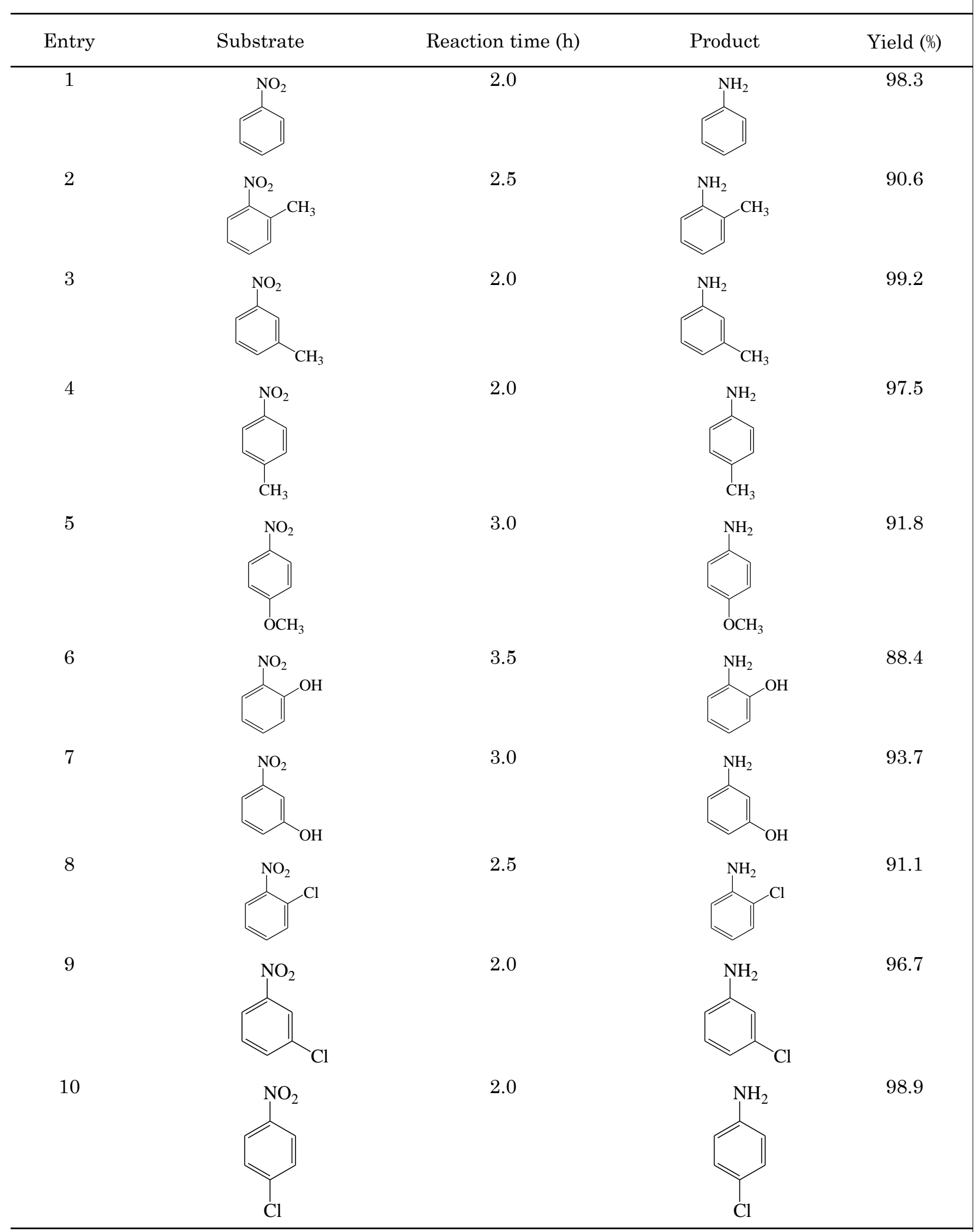

Reaction conditions: substrate $(10 \mathrm{mmol})$, sodium borohydride $(12 \mathrm{mmol})$, selenium $0.1 \mathrm{~g}$ and $\mathrm{AC} 0.5 \mathrm{~g}$ in ethanol/water reacting at room temperature. 
vorable for the reaction (entries $2,4,5,7,8,9$, 10). However, when the proportion of water is too high, the solubility of nitrobenzene will decrease and the yield of aniline will become low. From Table 2 we learn that the yield of aniline is the highest when the volume ratio of ethanol to water is three.

\subsection{Reusability}

At the end of catalytic reduction of nitrobenzene, air was introduced into the flask and the catalyst was precipitated and recovered. The catalyst was also tested for reusability and it was found that it could be reused without affecting the activity till at least four cycles (Table 3). Before the reaction, the selenium was solid in the mixture, which is different in phase with the reagents. During the reaction process, selenium reacts with sodium borohydride to form $\mathrm{H}_{2} \mathrm{Se}$ or its anions, which can dissolve in the solvent to form a homogeneous catalytic system. In the system, $\mathrm{H}_{2} \mathrm{Se}$ or its anions reduces nitrobenzene to aniline and is oxidized to selenium, so the catalytic activities of the recovered selenium are practically the same as those of the fresh catalysts [16].

\subsection{A Possible Reaction Pathway of Re- duction}

A reaction mechanism is proposed for the reduction of nitrobenzene as shown in Scheme 2 . It has been well documented that selenium reacts with reducing agent to form $\mathrm{H}_{2} \mathrm{Se}$ [17] which then promotes the reduction of nitroarenes absorbed in AC to nitrosobenzene and $N$ hydroxybenzenamine. Finally the $\mathrm{N}$ hydroxybenzenamine reduced rapidly by $\mathrm{H}_{2} \mathrm{Se}$ and water to afford the desired aniline.

\subsection{Reduction of Nitro Compounds}

To further explore the utility of this catalytic system several substituted nitroarenes were studied (Table 4). In all cases, amines were found to be the only product and the side products of nitro reduction were not observed in the final product. The method also shows promise for chemoselective reduction with high yield. Sensitive functional groups, such as: $-\mathrm{Cl}$, $-\mathrm{OH}$ and $-\mathrm{OCH}_{3}$, did not undergo any change under the reaction conditions. In most cases, the corresponding amines were obtained in excellent yields. It is worth mentioning here that the activity is significantly influenced by the position of the substituents on the aromatic ring. The presence of a group, such as: $-\mathrm{CH}_{3}$, $-\mathrm{OCH}_{3},-\mathrm{OH}$ or $-\mathrm{Cl}$, ortho to the nitro group, decreased the yield to a larger extent than at the para or meta-position due to steric effects. On the other hand, electron withdrawing/donating groups, such as: $-\mathrm{Cl},-\mathrm{CH}_{3}$, $-\mathrm{OCH}_{3},-\mathrm{OH}$, do not have a significant influence on the reaction.

\section{Conclusions}

At room temperature, selenium and ACcatalyzed reduction of nitroarenes to amines with sodium borohydride in ethanol and water. Under the optimized conditions, efficient and selective reduction of nitroarenes into the corresponding aromatic amines occurred over a recyclable selenium catalyst. The advantages of this method are: high selectivity, ease of operation, high yields of the amines and no equipment of pressure apparatus. The present method offers an economical, state and environmentally benign alternative to the currently available procedures.

\section{Acknowledgments}

The authors wish to acknowledge the financial support from Xuzhou Institute of Technology (XKY2012007).

\section{References}

[1] Yin, J.M., Zhang, R., Jia, Y.P., Cui, Y. N., Zhou, G.Y., Gao, D.B. (2010). Research Progress in Preparation of Aromatic Amine via Reduction of Aromatic Nitro Compounds. Chinese Chemical Research. 21(1): 96-101.

[2] Xu, S.L., Chen, H.B., Li, S.D. (2006). Progress in Preparation of Aromatic Amine from Aromatic Nitro Compounds by Catalytic Hydrogenation. Chinese Speciality Petrochemicals. 23(4): 58-61.

[3] Johnstone, R.A.W., Wilby, A.H., Entwistle, I.D. (1985). Heterogeneous Catalytic Transfer Hydrogenation and its Relation to other Methods for Reduction of Organic Compounds. Chemistry Review. 85(2): 129-170.

[4] Zeynizadeh, B., Setamdideh, D. (2006). $\mathrm{NaBH}_{4} /$ Charcoal: a new Synthetic Method for Mild and Convenient Reduction of Nitroarenes. Syn. Commun. 36: 2699-2704.

[5] Shil, A.K., Sharma, D., Guha, N.T., Das, P. (2012). Solid Supported $\operatorname{Pd}(0)$ : an Efficient Recycable Heterogeneous Catalyst for Chemoselective Reduction of Nitroarenes. Tetrahedron Lett. 53: 4858-4861.

[6] Sen, I.K., Maity, K., Islam, S.S. (2013). Green Synthesis of Gold Nanoparticles Using a Glucan of an Edible Mushroom and Study of Catalytic Activity. Carbohydrate Polymers. 91: 518-528. 
[7] Kiasat, A.R., Mirzajani, R., Ataeian, F., Fallah-Mehrjardi, M. (2010). Immobilized Silver Nanoparticles on Silica Gel as an Efficient Catalyst in Nitroarene Reduction. Chinese Chem. Lett. 21: 1015-1019.

[8] Liu, X., Cheng, H., Cui, P. (2014). Catalysis by Silver Nanoparticles/Porous Silicon for the Reduction of Nitroaromatics in the Presence of Sodium Borohydride. Appl. Surf. Sci. 292: 695-701.

[9] Pogorelić, I., Filipan-Litivić, M., Merkaš, S., Ljubić, G., Capanec, I., Litivić, M. (2007). Rapid, Efficient and Selective Reduction of Aromatic Nitro Compounds with Sodium Borohydride and Raney Nickel. J. Mol. Catal. A-Chem. 274: 202-207.

[10] Nandanwar, S. U., Chakraborty, M. (2012). Synthesis of Colloidal $\mathrm{Cu} / \gamma-\mathrm{Al}_{2} \mathrm{O}_{3}$ by Microemulsion and Catalytic Reduction of Aromatic Nitro Compounds. Chin. J. Catal. 33(9): 1532-1541.

[11] Jayabal, S., Ramaraj, R. (2014). Bimetallic $\mathrm{Au} / \mathrm{Ag}$ Nanorods Embedded in Functionalized Silicate Sol-Gel Matrix as an Efficient Catalyst for Nitrobenzene Reduction. Appl. Catal. A-Gen. 470: 369-375.

[12] Pradhan,, N., Pal, A., Pal, T. (2002). Silver Nanoparticle Catalyzed Reduction of Aromatic Nitro Compounds. Colloid Surf. APhysicochem. Eng. Asp. 196: 247-257.
[13] Vadakkekara, R., Chakraborty, M., Parikh, P. A. (2012). Reduction of Aromatic Nitro Compounds on Colloidal Hollow Silver Nanospheres. Colloid Surf. A-Physicochem. Eng. Asp. 399: 11-17.

[14] Zhang, X.P., Miao, J.H., Sun, Y.B. (2009). Recent Progress in Selenium-Catalyzed Reactions. Chin. J. Org. Chem. 29: 1555-1563.

[15] He, G.Y., Liu, W.F., Sun, X.Q., Chen, Q., Wang, X., Chen, H.Q. (2013). $\mathrm{Fe}_{3} \mathrm{O}_{4}$ Graghene Oxide Composite: A Magnetically Separable and Efficient Catalyst for the Reduction of Nitroarenes. Mater. Res. Bull. 48: 1885-1890.

[16] Liu, X.Z., Lu, S.W. (2001). Selective Formation of Aromatic Amines by Selenium-Catalyzed Reduction of Aromatic Nitro Compounds with $\mathrm{CO} / \mathrm{H}_{2} \mathrm{O}$ under Atmosspheric Pressure. J. Mol. Catal. A-Chem. 212: 127-130.

[17] Li, J., Qu, Y.Q., Han, K.L, Lu, S.W., He, G.Z. (2009). Formation Mechanism of Selane from the Reaction System of Selenium, Carbon Monooxide and Water. Chem. J. Chinese U. 27(4): 708-710. 